\title{
Nonlinear dynamic behaviors of outer shell and upper deck structures subjected to impact loading in maritime environment
}

https://doi.org/10.1515/cls-2019-0012

Received May 07, 2019; accepted Jul 02, 2019

Abstract: Ship collision appears as the most threatening loading accounting for structural casualties and numbers of casualties after impact on the target ship. In order to avoid such losses against collision, better safety during activities in maritime environment is demanded. Therefore, assessment of ship structure is needed to understand dynamic effect of the impact and quantify nonlinear behavior of local members. This study is conducted to achieve those aims by deploying nonlinear finite element analysis (NLFEA) to idealized ship collision event. Validation of the numerical method is performed by comparing results of a modeled collision case with various empirical calculations. Design for impact loading in main analysis considers side collision to main hull structure, which single side skin (SSS) and double side skin (DSS) types are modeled. Investigation is also directed to influence of the target members on the main hull to capacity of absorbed energy and characteristic of structural resistance. Analysis results indicate that good understanding is successfully obtained in terms of structural damage-energy relation. Confirmation of the current calculation using numerical calculation is also confirmed considering the modeled cases and empirical results agree well. Tendency of hull responses concluded that the longitudinal members contribute more to structural resistance against side collision.

Keywords: ship-ship collision, local member effects, absorbed energy, crushing force, NLFEA techniques

\footnotetext{
*Corresponding Author: Aditya Rio Prabowo: Department of Mechanical Engineering, Sebelas Maret University, Surakarta 57126, Indonesia; Email: aditya@ft.uns.ac.id

Jung Min Sohn: Department of Naval Architecture and Marine Systems Engineering, Pukyong National University, Busan 48513, South Korea
}

\section{Introduction}

Ship is initially built for human to interact with his fellow who stays in different islands, and engage in trade for various goods with other countries. Most ships in the past are designed as merchant ship to support these activities, which as further development rate of naval technology, design of the merchant ship begins to change to fulfil demand for specific cargo, such as passenger, oil and chemical compounds, nuclear resource etc.

During its operation in maritime environment, the developed ship and carrier are frequently encountering risk of impact loading, resulting immense losses in terms of ship structure, marine ecosystem, industrial economy and human safety. Immense damage is expected as ship in this era, even though it has been developed, since consideration to reduce effect of impact loading still conventional. Numbers of marine accidents cause massive causalities due to lack of crashworthiness knowledge in ship design, such as the Titanic in 1912 and the Exxon Valdez in 1989. These accidents which are caused by impact loading, later trigger global revolution in marine activity regulations, i.e. Safety of Life at Sea (SOLAS) and Oil Pollution Act (OPA) are taken into force for various naval and carrier. Furthermore, Common Structural Rules (CSR) for bulk carrier and container ship are also stating that double skin structure (DSS) is highly recommended. Besides these efforts, development in navigational technology and seamanship requirement is also taken as active method to reduce risk of impact loading in maritime environment, such as collision and grounding $[1,2]$.

However, occurrence of collision and grounding is still main concern as these events contribute to main cause of ship losses for last decade. More than 300 cases are recorded for collision and grounding or approximately $26 \%$ of all loss causes. This number is increasing to be more than $90 \%$ if other form of impact loading, explosion, is included together with chain effect of impact, such as foundering and hull damage [3]. Based on these data, it can be concluded that collision and its casualty loss still

¿ Open Access. (C) 2019 A. R. Prabowo and J. M. Sohn, published by De Gruyter Attribution 4.0 License 
occur despite of advance development of marine regulation and active attempt to avoid such event in ship operational. Its characteristic as dynamic - nonlinear phenomenon makes collision, including grounding and explosion have wide range of phenomenon scenario to be subject for sustainable research regarding structural safety and damage assessment in maritime environment. Implementation of the advance structural type to avoid damage on cargo and hull as well as to replace conventional single side skin (SSS), are already massively used in shipbuilding industry. Numbers of institutional research related to impact loading and ship structure have been conducted, such as hull girder energy by Pedersen and Li [4], icetanker collision by Gagnon and Wang [5], ship impact to offshore structure by Fan and Yuan [6], hull tanker under minor collision by Liu and Soares [7], effect of rebounding phenomenon by Prabowo et al. [8], continuum damage mechanics by Martinez et al. [9], design principles and criteria by Collette et al. [10], and hazard identification of accidental loading by Youssef and Paik [11]. Presently, however, there is a scant research related to explicit assessment of SSS and DSS which is directed to measure structural performance against impact loading during ship operation.

Considering the mentioned trend, this work aims to conduct a series of numerical analysis to observe dynamic behavior of local members on SSS and DSS under impact loading. Calculation is performed using nonlinear finite element analysis (NLFEA) technique, which configuration of the analysis is validated by empirical calculation of the developed formulae of absorbed energy. Designed scenarios adopt ship-to-ship collision as representative of impact loading in maritime environment. Results of the calculation and assessment are expected to be a useful and reliable reference in designing hull arrangement accounting for extreme condition during preliminary design phase.

\section{Characteristic of analysis and instrument}

Ship collision considered in this work is categorized into nonlinear dynamic phenomenon which needs suitable calculation strategy to solve its almost-limitless scenario. Nonlinear is embedded in analysis as fundamental assumptions of linear analysis are violated due to material and structure exceed their yield limit. In this state, both components (involved ships) experience large extent of deformation, which may occur as tearing, crushing and folding. In other hand, dynamic characteristic is considered as calculation results will change progressively dependent to time. To solve phenomenon with these criteria, explicit strategy is preferred since several assumptions are advantageous, i.e.:

1. Simple inversion is needed as the subject is a lumped mass matrix

2. Mathematical equation is uncoupled and can be solved directly

3. Due to characteristic in no. 2, convergence check is not needed

4. Inversion on the stiffened matrix is not required which all nonlinear parameters (including contact) are include in the internal force vector

5. Very small time step is required to maintain stability limit which is match with small size of time process of ship collision (usually less than $1 \mathrm{sec}$.).

$$
\begin{gathered}
\left\{\boldsymbol{a}_{t}\right\}=[\boldsymbol{M}]^{-1}\left(\left\{\boldsymbol{F}_{t}^{\text {ext }}\right\}-\left\{\boldsymbol{F}_{t}^{\text {int }}\right\}\right) \\
F^{i n t}=\sum\left(\int_{\Omega}\left(B^{T} \sigma_{n} d \Omega+F^{h g}\right)+F^{c o n t}\right) \\
\left\{\boldsymbol{v}_{t+\Delta t / 2}\right\}=\left\{\boldsymbol{v}_{t-\Delta t / 2}\right\}+\left\{\boldsymbol{a}_{t}\right\} \Delta t_{t} \\
\left\{\boldsymbol{u}_{t+\Delta t}\right\}=\left\{\boldsymbol{u}_{t}\right\}+\left\{\boldsymbol{v}_{t+\Delta t / 2}\right\} \Delta t_{t+\Delta t / 2} \\
\left\{\boldsymbol{x}_{t+\Delta t}\right\}=\left\{\boldsymbol{x}_{0}\right\}+\left\{\boldsymbol{u}_{t+\Delta t}\right\}
\end{gathered}
$$

where: $\left\{\boldsymbol{a}_{t}\right\}$ is the acceleration; $\left\{\boldsymbol{F}_{t}^{e x t}\right\}$ is the applied external and body force vector; $\left\{\boldsymbol{F}_{t}^{\text {int }}\right\}$ is the internal force vector; $F^{\text {hg }}$ is the hourglass resistance force; $F^{\text {cont }}$ is the contact force; $B^{T}$ is the form identical to the linear discrete strain-displacement matrix; $\Omega$ is the solid volume; $\sigma_{n}$ is the internal stress; $\left\{\boldsymbol{v}_{t}\right\}$ is the velocity at time $t ;\left\{\boldsymbol{u}_{t}\right\}$ is the displacement at time $t ;\left\{\boldsymbol{x}_{0}\right\}$ is the initial geometry; $\left\{\boldsymbol{x}_{t}\right\}$ is the updated geometry at time $t ; \Delta t_{t}$ is the difference in time of at time $t$ compared to the initial/selected condition.

Nonlinear dynamic analysis of the designed ship collision is performed using ANSYS LS-DYNA [12], and the implemented algorithm of the explicit strategy is characterized in Equation 1 for acceleration component by a use of a central difference time integration. Component of the internal force is expanded further in Equation 2 in order to classify nonlinear parameters. After accelerations are evaluated, the velocities and displacements are then given in Equations 3 and 4, consecutively. The updated state of geometry is presented as combination of the displacement increments and initial state of geometry (see Equation 5). Stability limit in explicit strategy will be stable if the time step size is smaller than the critical time step size, which is very suitable for small transient. 
Computational instrument to analyze a series of collision problem is given as follows: 4th Generation Intel Core i7-4790 Processor ( 4 cores, 8 threads, $3.60 \mathrm{GHz}$ processor base frequency, $84 \mathrm{~W}$ TDP) $4.00 \mathrm{GHz}, 120 \mathrm{~Gb} \mathrm{SSD}$ (SATA 6GB/sec interface, $540 \mathrm{MB} / \mathrm{sec}$ sequential read), 16 GB RAM. Approximation of the computing power: $R_{\text {percore }}$ $=4.13$ Gflops and $R_{\text {percomputer }}=32.68$ Gflops.

\section{Literature review on marine accidents and pioneer works}

Developments of shipbuilding industry has reached massive range which have considered various aspects in shipping, including serviceability, safety, economy, and sustainability to keep moving forward. Reason behind such positive tendency is seaborne trade which continues to grow fast as affected by both bilateral and multilateral freetrade agreement (FTA) of countries across the globe. Increment in shipbuilding production due to high demand of marine carrier and vessel inevitably causes high traffic in international and local shipping routes, waterway/canal, and other shipping environment. This situation makes risk of accidental event tends to occur, such as due to human error, navigation failure, loss control, which impact loading appears as the biggest threat among of all accidental events in maritime area. In the recent decade itself, 2012 is remembered as grieve years for shipbuilding and shipping parties as tragic loss of Costa Concordia-Italy, in January is followed by sinking of Rabaul Queen-Papua New Guinea in February. It is interesting enough considering the year coincides with 100 year since one of the biggest marine disaster in history of cruise and passenger vessel, TitanicUK, which collided with an iceberg in North Atlantic and caused approximately 1500 human life losses. Authorities and industrial parties were conducting sustainable effort to avoid such disaster, or at least reduce its casualties. Vision regarding human role in shipping are shifted according to Allianz Global Corporate \& Specialty (ACGS), which attempt to provide training and ship for crew and seafarer in both shipping and marine industries are no longer sole priority. The important task now is implementing a culture of safety with support of the sharpest weapons in form of regulation to improve safety [13], such as:

1. International Convention for the Safety of Life at Sea (SOLAS), 1974, as amended

2. International Convention for the Prevention of Pollution from Ships, 1973, as modified by the Protocol of 1978 relating thereto and by the Protocol of 1997 (MARPOL)

3. International Convention on Standards of Training, Certification and Watchkeeping for Seafarers (STCW) as amended, including the 1995 and 2010 Manila Amendments

4. Convention on the International Regulations for Preventing Collisions at Sea (COLREG), 1972

5. Convention on Facilitation of International Maritime Traffic (FAL), 1965

6. International Convention on Load Lines (LL), 1966

7. International Convention on Maritime Search and Rescue (SAR), 1979

8. Convention for the Suppression of Unlawful Acts Against the Safety of Maritime Navigation (SUA), 1988, and Protocol for the Suppression of Unlawful Acts Against the Safety of Fixed Platforms located on the Continental Shelf (and the 2005 Protocols)

9. International Convention for Safe Containers (CSC), 1972

10. Convention on the International Maritime Satellite Organization (IMSO C), 1976

11. The Torremolinos International Convention for the Safety of Fishing Vessels (SFV), 1977

12. International Convention on Standards of Training, Certification and Watchkeeping for Fishing Vessel Personnel (STCW-F), 1995

13. Special Trade Passenger Ships Agreement (STP), 1971 and Protocol on Space Requirements for Emergency Position Indicating Radio Beacon Special Trade Passenger Ships, 1973

Improvement and implementation of maritime regulation cannot be separated from research and assessment contributions of university and institution scholars who have taken impact loading as their well-grounded study agenda for more than five decades. Initial concern of impact loading on marine structures is addressed by $\mathrm{Mi}$ norsky [14] who took nuclear-powered ship as potential medium of massive environmental damage if leakage occurred on the reactor due to collision. A series of actual collision cases were summarized to introduce a mathematical expression (Equations 6 and 7) for rapid estimation of absorbed energy accounting for damage volume. This research later was refined by Woisin [15] who assumed the previous formula was only suitable for highenergy collision. Therefore, he composed another expression (Equation 8) which developed constant value in Minorsky expression. Research trends of this topic is developed steadily until the end of 1990, which remarkable evolution of computational technology makes finite element 
method (FEM) is used to calculate the nonlinear phenomena. Due to large extent of scenario possibility, role of the FEM is very assisting in validation and even expanding case study for other calculation methodology, such as analytical strategy which considers upper-bound theorem and experimental test.

$$
\begin{gathered}
E_{M}=47.2 R_{T}+32.7 \\
R_{T}=\sum P_{N} L_{N} t_{N}+\sum P_{n} L_{n} t_{n} \\
E_{W}=47.2 R_{T}+0.5 \sum\left(h . t_{s}^{2}\right) \\
E_{z-\text { ten }}=0.77 \epsilon_{c} \sigma_{0} R_{T} \\
E_{z-\text { crush }}=3.50\left(\frac{t}{d}\right)^{0.67} \sigma_{0} R_{T} \\
E_{z \text {-tear }}=3.21\left(\frac{t}{l}\right)^{0.6} \sigma_{0} R_{T}
\end{gathered}
$$

where: $R_{t}$ is the destroyed material volume of the involved structures/entities in impact, or so called resistance factor; $E_{M}$ is the absorbed energy of the Minorsky for high-energy collision; $P_{N}$ and $P_{n}$ are the depth of occurred damage on the striking ship and struck ship respectively; $L_{N}$ and $L_{n}$ are the length of damage on the striking ship and struck ship members; $t_{N}$ and $t_{n}$ are the thickness of damage in on the striking ship and struck ship members; $E_{W}$ is the absorbed energy of the Woisin for low-energy collision; $h$ is the height of the destroyed or heavily deformed longitudinal members; $t_{s}$ is the thickness of the members; $\sigma_{0}$ is the flow stress of the material; $E_{z-t e n}$ is the absorbed energy of the Zhang for plastic tension damage mode; $E_{z-c r u s h}$ is the absorbed energy of the Zhang for crushing damage mode; $E_{z \text {-tear }}$ is the absorbed energy of the Zhang for tearing damage mode; $\epsilon_{c}$ is the ultimate strain of the applied material; $t$ is the average thickness of the destroyed members; $d$ is the average width of the plate in the crushed cross-section; $l$ is the tearing length.

Collaboration of these methods are used widely, e.g. in progressive structural crashworthiness, resistance to penetration of stiffened plates, application of super-element strategy, assessment of innovative structure members, analytical in raked bow collisions, damage characteristics caused by ice collision [16], structural responses of doublebottom structures in shoal grounding, effectiveness of thin-walled hull [17], risk assessment of striking ship impacts [18], effects of selected collision's parameters [19] and progressive failure under side-bow collisions [20].
Long journey of these collaborations and sustainable efforts in accidental events introduces a latest estimation formula for rapid calculation of absorbed energy. Compared to his pioneers, scholars classify the damage pattern into several modes, and compose specific formula for them. As presented in Equations 9-11, they stand for plastic tension [21], crushing damage [21] and tearing damage [22].

\section{Evaluating methodology of the numerical setting and calculation}

\subsection{Brief description and analysis configuration}

A reference of collision case is adopted to validate configuration of the current NLFEA technique and configuration, which later will be used to solve a series of impact loading problems. The case took place in Indonesia water territory in May 2014, on the Sunda strain between Sumatra and Java. Location of the incident is included in international shipping route for Singapore Port, Singapore-Fremantle Port, Australia, and also in inter-island route for passenger and Ro-Ro carriers with very high traffic, operates $24 / 7$. In this very cross line of two routes, a $144 \mathrm{~m}$ reefer cargo vessel hit-and-run a $85 \mathrm{~m}$ passenger carrier, and left structural casualties on the carrier's side skin with size $7 \mathrm{~m}$ in length.

Investigation and reparation of the carrier were immediately performed, which concluded the tearing on the skin reached approximately $2.5 \mathrm{~m}$ in depth and $5 \mathrm{~m}$ in width. Checking and benchmarking of the numerical methodology in this research will involve collaboration of three strategies, namely data of actual collision which is obtain by field survey [23], NLFEA, and empirical calculation. In the first stage, impact phenomenon of this case is modeled by a use of finite element method, which considers three impact targets on the side skin of the struck ship (passenger carrier), namely middle deck, upper deck and outer shell. Velocity during impact by the striking bow of the cargo reefer is defined as 12 knot or approximately equal with $6.17 \mathrm{~m} . \mathrm{s}^{-1}$ which is assumed based on the service speed of the ship. Validation of the calculated energy in NLFEM is compared with formula of three pioneers which the mathematical expressions have been presented in prior discussion. Results of the analysis in terms of damage pattern will be summarized in the second stage to compare them to the actual damage data obtained in field. 


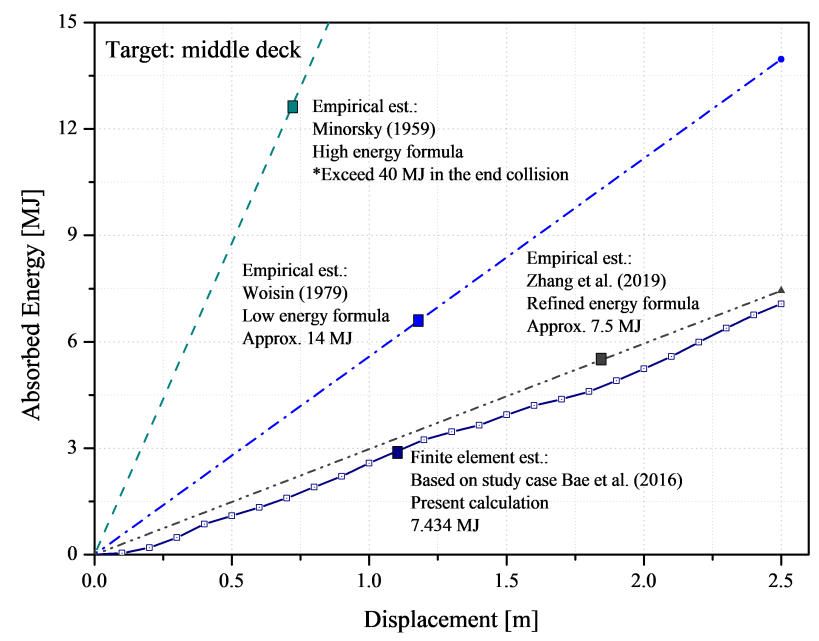

(a) Target: middle deck

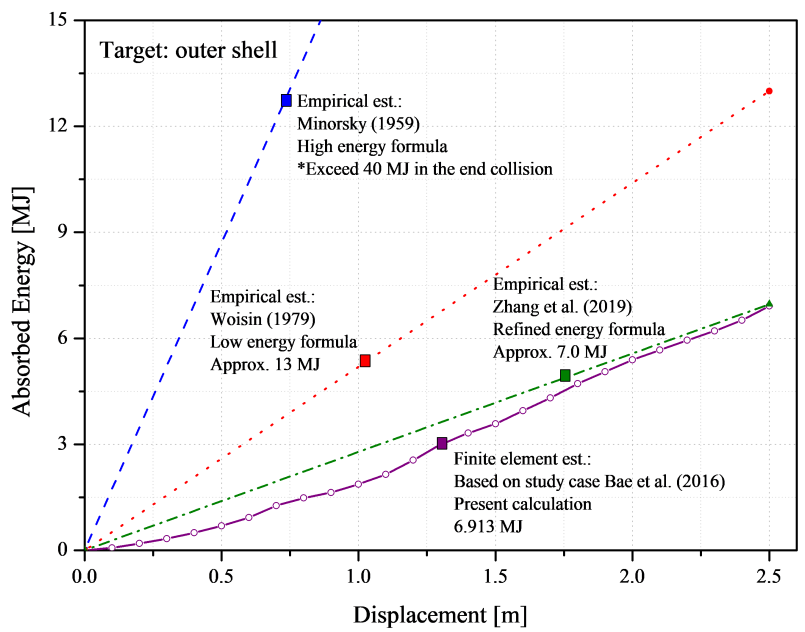

(c) Target: outer shell

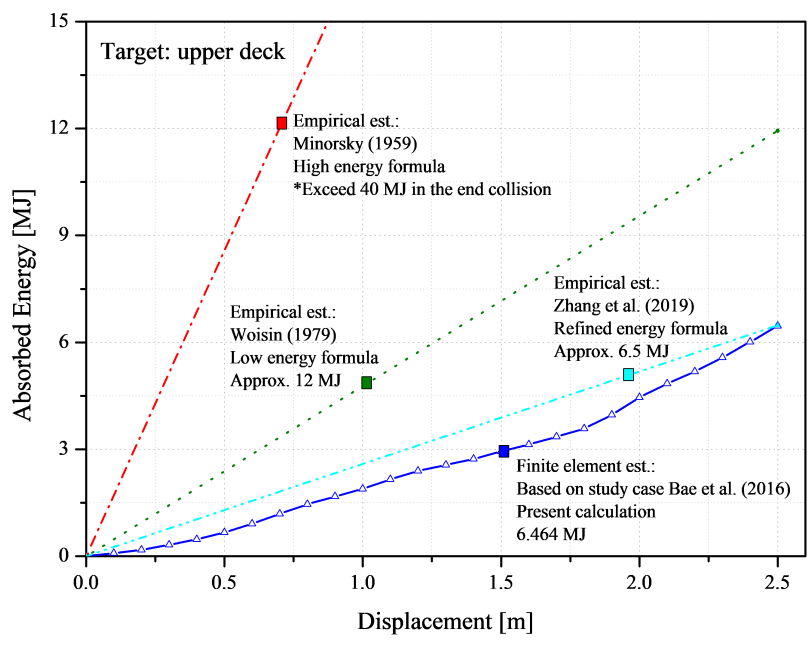

(b) Target: upper deck

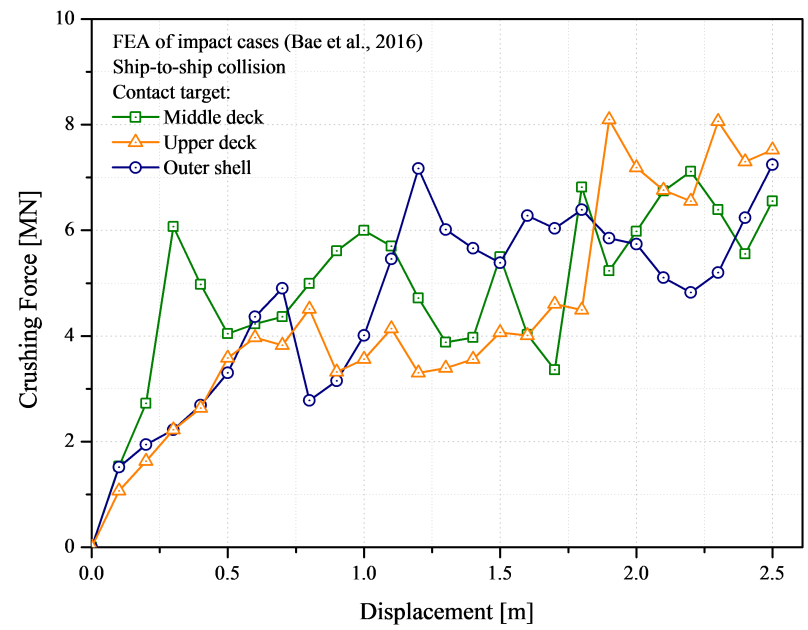

(d) Crushing force of the three targets

Figure 1: Comparison of the analyzed collision scenario by NLFEA and empirical estimation

\subsection{Results}

Investigation in terms of the absorbed energy in Figure 1 concludes that the calculated collision problems using NLFEA reaches good agreement with empirical estimation. Based on comparison with three empirical expressions, the recent developed formula by Zhang is the most similar with numerical results.

This results in same time also verify characteristic of two other formulae, namely Minorsky and Woisin. The Minorsky mathematical form produced very high energy estimation which approximately more than $40 \mathrm{MJ}$ for each case. It is predicted that the constant value of the Minorsky is quite large, and makes result of ship collision in the present case is overestimated. This sentence is supported by result behavior of the Woisin formula which the con- stant value of the first formula is refined, shows more reasonable value and closer to Zhang estimation. Taking results of the other work conducted by Ozguc et al. [24] into this discussion, are suggesting that Minorsky formula produced more similarity than the current work, however, subject of the side skin structure in the work was larger with overall ship length $279.91 \mathrm{~m}$ and $279.29 \mathrm{~m}$ for single side skin and double side skin structure, consecutively. This tendency shows that larger the involved ship in impact, it can be collision and grounding, the evaluated energy from the finite element analysis will be more similar to Minorsky estimation. Besides the energy, results of the crushing force is also given in this discussing. It is obtained that the deck members produced higher force increment than the shell during damages were expanding. 
Table 1: Summary of the damage pattern of the analyzed collision problems

\begin{tabular}{|c|c|c|c|c|c|c|}
\hline \multirow{2}{*}{$\begin{array}{l}\text { Target on } \\
\text { local } \\
\text { members }\end{array}$} & \multicolumn{2}{|c|}{ Casualties size } & \multicolumn{4}{|c|}{ Damage pattern accounting for structural members } \\
\hline & $\begin{array}{l}\text { Long. length } \\
\qquad-L[\mathrm{~m}]\end{array}$ & $\begin{array}{l}\text { Ver. width - } \\
\quad \text { W [m] }\end{array}$ & Outer shell & $\begin{array}{c}\text { Transverse } \\
\text { frame }\end{array}$ & Middle deck & Upper deck \\
\hline Middle deck & 4.20 & 5.00 & $\begin{array}{l}\text { Long. tearing } \\
\text { and ver. } \\
\text { tearing under } \\
\text { middle deck }\end{array}$ & $\begin{array}{c}\text { Rupture } \\
\text { (under middle } \\
\text { deck) }\end{array}$ & $\begin{array}{c}\text { Crushed (edge } \\
\text { to middle } \\
\text { parts) }\end{array}$ & $\begin{array}{c}\text { Crushed (edge } \\
\text { part) }\end{array}$ \\
\hline Upper deck & 7.00 & 3.00 & $\begin{array}{l}\text { Ver. tearing } \\
\text { between } \\
\text { upper and } \\
\text { middle decks }\end{array}$ & $\begin{array}{c}\text { Rupture } \\
\text { (between } \\
\text { upper and } \\
\text { middle decks) }\end{array}$ & $\begin{array}{l}\text { Indented } \\
\text { (edge part) }\end{array}$ & $\begin{array}{c}\text { Crushed (edge } \\
\text { part) }\end{array}$ \\
\hline Outer shell & 7.00 & 4.50 & $\begin{array}{l}\text { Long. tearing } \\
\text { and ver. } \\
\text { deformation } \\
\text { due to penet. } \\
\text { of striking bow }\end{array}$ & $\begin{array}{c}\text { Rupture } \\
\text { (between } \\
\text { upper and } \\
\text { middle decks) }\end{array}$ & $\begin{array}{l}\text { Indented - } \\
\text { minor crush } \\
\text { (edge part) }\end{array}$ & $\begin{array}{l}\text { Crushed (edge } \\
\text { part) - long. } \\
\text { deformation } \\
\text { to counter } \\
\text { direction of } \\
\text { penetration }\end{array}$ \\
\hline Collision ref. & 7.00 & 5.00 & $\begin{array}{l}\text { Long. tearing } \\
\text { and ver. } \\
\text { deformation } \\
\text { due to penet. } \\
\text { of striking bow }\end{array}$ & $\begin{array}{l}\text { Rupture } \\
\text { (between } \\
\text { upper and } \\
\text { middle decks) }\end{array}$ & $\begin{array}{l}\text { Indented - } \\
\text { minor crush } \\
\text { (edge part) }\end{array}$ & $\begin{array}{l}\text { Crushed (edge } \\
\text { part) - long. } \\
\text { deformation } \\
\text { to counter } \\
\text { direction of } \\
\text { penetration }\end{array}$ \\
\hline
\end{tabular}

Data of the casualty and damage pattern calculated by NLFEA is summarized in Table 1 and Figure 2. Striking bow impact to the middle deck produced damage length lesser than other targets, approximately $40 \%$ even though the damage width agreed well with the collision reference in 2014. On the other hand, the upper deck and outer shell showed good correlation in terms of the longitudinal length, with the most similar damage in terms of the vertical width is the outer shell with difference to the referred case $0.50 \mathrm{~m}$.

Impact to the upper deck is the second similar to the actual case in size, however, damage pattern on the outer shell and upper deck showed different pattern. Therefore, considering both damage size and pattern, target outer shell is the most similar. Concise conclusion of the discussion regarding the evaluated energy and damage characteristic, can be stated that the present numerical methodology is well enough to produce similar result compared to actual phenomenon. Configuration and setting in the initial assessment will be implemented in to extended investigation of nonlinear dynamic behavior of structural members under impact loading, ship-to-ship collision.

\section{Ship structures}

\subsection{Finite element model}

Involved ships in the designed collision problems use same vessel as deployed in the benchmark evaluation. The struck ship is the 683 DWT passenger carrier with principal dimension length between perpendicular $L p p=78 \mathrm{~m}$; breadth $B=15 \mathrm{~m}$; draft $T=4.3 \mathrm{~m}$ and height $H=10.4 \mathrm{~m}$. The striking ship is idealized based on the 7337 DWT cargo reefer with principal dimension: length over all $L o a=144.5$ $\mathrm{m}$; breadth $B=19.8 \mathrm{~m}$; draft $T=5.6 \mathrm{~m}$ and height $H=10.2$ $\mathrm{m}$. Geometrical models of the both ships are presented in Figure 3, which the struck ship is modelled based on single side skin (SSS) and double side skin (DSS) while the striking ship is focused on the forward part of the ship hull. The numerical geometry on the NLFEA is built based on shell element formulation Belytschko-Tsay which its application is considered suitable for nonlinear dynamic phenomenon, especially collision which produces significant geometrical change in very short time period (usually less than 1 sec.).

Material embedded on the structures is formulated in NLFEA using plastic-kinematic model with strain-rate de- 


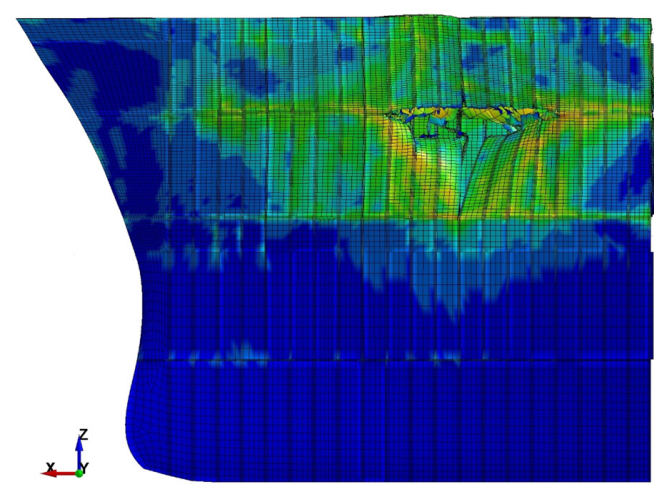

(a) Impact target 1: middle deck
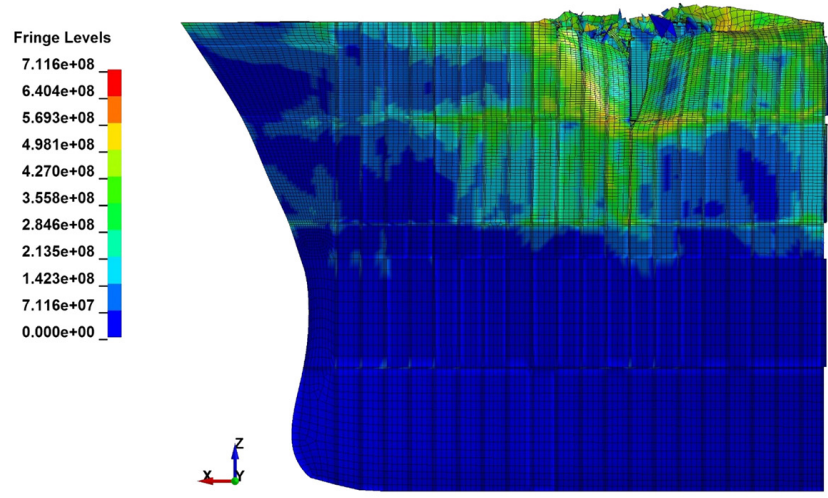

(b) Impact target 2: upper deck

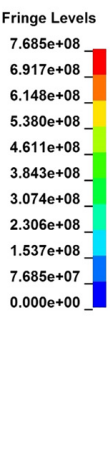
$6.148 \mathrm{e}+08$

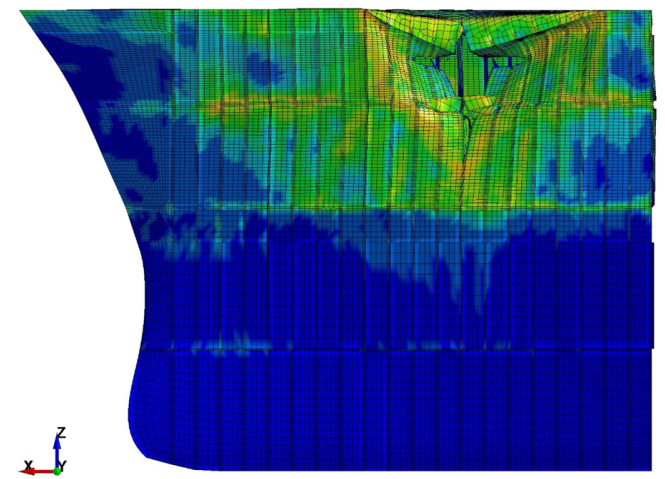

Fringe Levels

$7.127 \mathrm{e}+08$

$6.415 \mathrm{e}+08$

$5.702 \mathrm{e}+08$

$4.989 \mathrm{e}+08$

$4.276 \mathrm{e}+08$

$3.564 \mathrm{e}+08$

$2.851 \mathrm{e}+08$

$2.138 \mathrm{e}+08$

$7.127 \mathrm{e}+07$

$0.000 \mathrm{e}+00]$

(c) Impact target 3: outer shell

Figure 2: Structural conditions with v-m stress contours after three targets are given impact loading

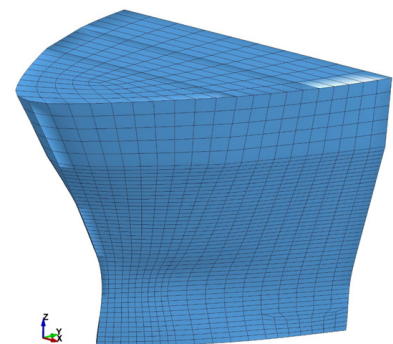

(a) Striking ship

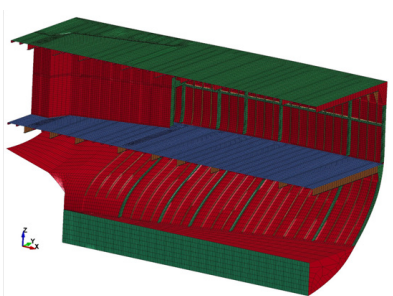

(b) Single side skin [SSS]

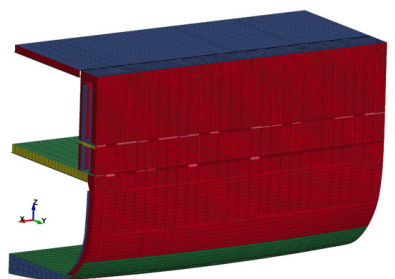

(c) Double side skin [DSS]

Figure 3: Idealized geometrical model for collision analysis

Table 2: Material model in collision analyses

\begin{tabular}{lc}
\hline Property & Value \\
\hline Density $\left[\mathrm{kg} \cdot \mathrm{m}^{-3}\right]$ & 7850 \\
Young modulus [MPa] & 210000 \\
Poisson ratio [-] & 0.3 \\
Yield strength [MPa] & 370 \\
Hardening parameter [-] & 0 \\
Strain rate value I [s $\left.{ }^{-1}\right]$ & 3200 \\
Strain rate value II [-] & 5 \\
Failure strain [-] & 0.2 \\
\hline
\end{tabular}

pendent concept (see Table 2). As described before that the impact loading, namely collision in this work, will cause high nonlinear behavior as it involves crushing, tearing and plastic tension on the local members. Therefore, it is a necessary to define failure condition of the structure. The failure of structure (local member) during impact loading takes place on the struck ship is a state where they surpassed the ultimate strain. Besides failure state, dis- 
cretizing large model such as ship structure in finite element technique is a crucial stage as large mesh element will lead to failure of the capture local deformation and response contour, even though calculation time can be reduce greatly. On contrary, too small mesh will cause unsatisfactory in forms of immense calculation time. Mesh size on the geometry will be set to follow element-length-tothickness (ELT) ratio in range 5-10 which has been verified in several impact problems, such as collision, stranding and raking [25]. The area on the impact target, such as upper side hull, will be applied with mesh size with smaller ratio while farther form the target, larger mesh ratio will be applied as no significant damage nor response contour is expected in the area.

\subsection{Impact scenario and boundary condition}

Collision problem in this work is assumed as interaction between two ships, which the striking ship impacts the side hull of the struck ship. This phenomenon is modelled as surface-to-surface contact in numerical configuration. The kinematic and static values of friction coefficient are given as $\mu_{k}=0.57$ and $\mu_{s}=0.74$ consecutively. External dynamic criterion, namely collision angle is set to be $90^{\circ}$ so that two ships form T-collision. During collision takes place, the striking ship is given by uniform velocity $V=$ $6.17 \mathrm{~m} \cdot \mathrm{s}^{-1}$ to move to several targets on the single side skin (SSS) and double side skin (DSS) structures. On geometry of the striking ship, rigid element are attached, which leads to damage occurrence is expected exclusively on the target. The impact targets of the striking ship are directed to the upper deck and outer shell/skin of the struck ship. On the other hand, the struck ship (SSS and DSS) is set to be fix on the centerline. They are restrained from axial and rotational displacements, which the boundary is placed on the end of model, specifically on transverse frame, longitudinal deck and inner bottom shell.

\section{Results and discussion}

\subsection{Absorbed energy}

The absorbed energy reflects amount of energy which is needed to severely damage or even destroy the involved entities during any kind of impact phenomenon. The energy is one of important crashworthiness criteria, which is usually used in assessment. In this work, this criterion is presented to acquire disparity between structural perfor-

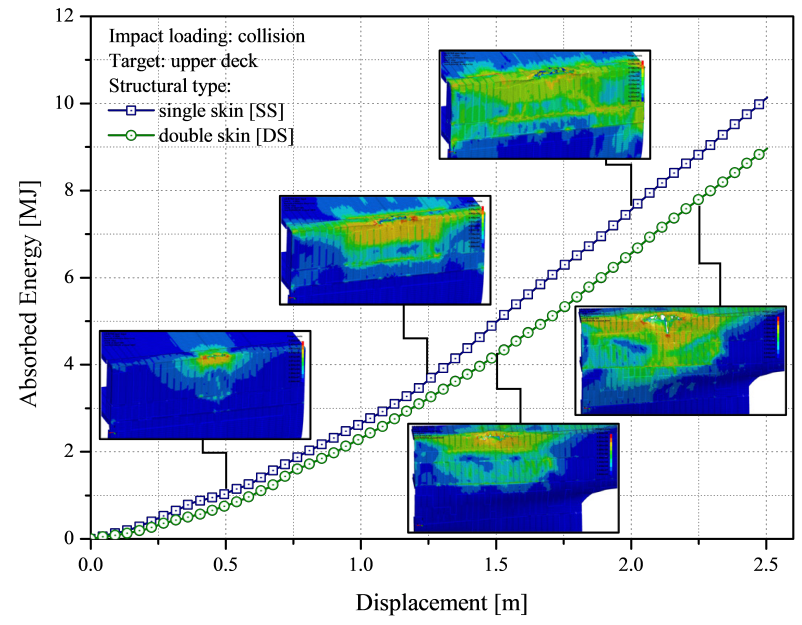

(a) Target: upper deck

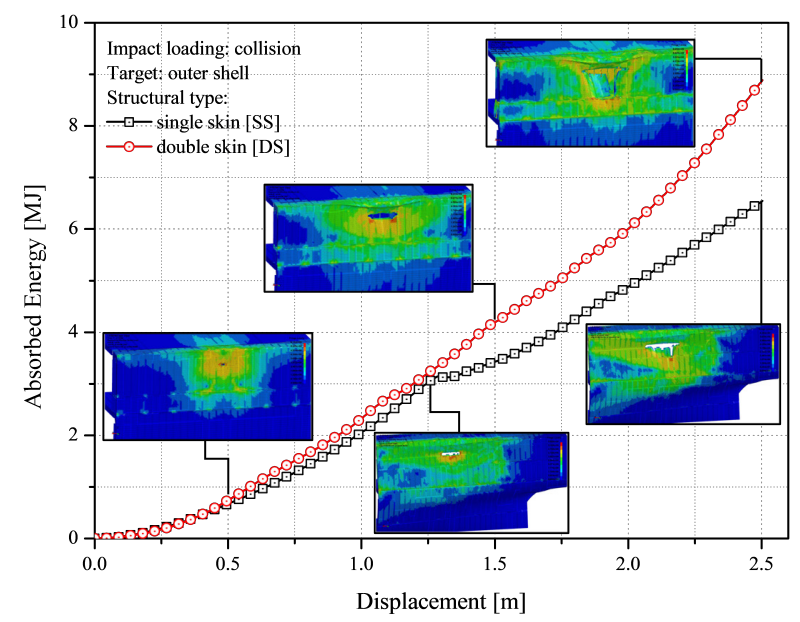

(b) Target: outer shell

Figure 4: Results of the absorbed energy for two targets

mances against side collision, including their members. As presented in Figure 4, it can be seen that the smooth and similar tendency during contact to the upper deck is obtained for both SSS and DSS. It marks an indication that change in structural types is not directly proportional to change of the absorbed energy under impact. Confirmation of the energy is shown by similarity in progressive damage pattern, which high difference is not found in impact targeted to the upper deck.

Observation is continued to the outer shell, which presented quite difference after displacement surpassed $1.25 \mathrm{~m}$. This tendency occurred as the stringer which supports the SSS has failed in resisting bow penetration. Energy of the DSS still steadily increased as the inner skin was remain intact and acted as member which provided resistance to penetration. In terms of the DS, it is con- 

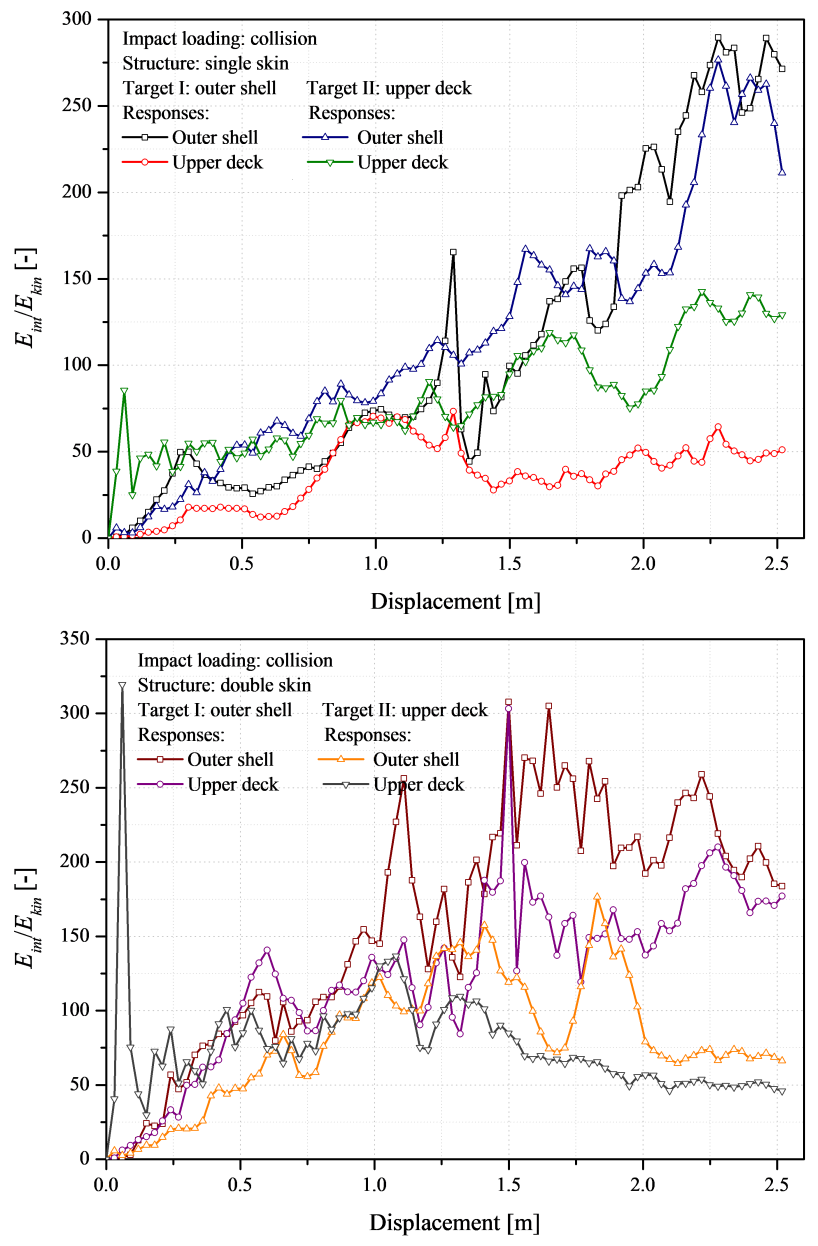

Figure 5: Summary of energy ratio for each impact target

cluded that change in structural types is significantly affect amount of the absorbed energy with approximation $30 \%$.

Related to the energy, behavior of the damage is also possibly traced and quantified by observing energy ratio. This ratio represents comparison between internal (absorbed) energy and kinetic energy. During impact took place on the SSS, both targets indicated that the most severe damage was experienced by the outer shell with quite large disparity in the end of the impact. However, different tendency is shown during assessment on the DSS. The ratio showed that impact on the Target I (outer shell) overwhelmed the Target II (upper deck). To explain this phenomenon, result of previous observation has to be accounted. It is found previously that the most contribute member in side collision is the outer shell. Therefore, the Target II produced lower ratio is caused by less damage occurred during side collision.
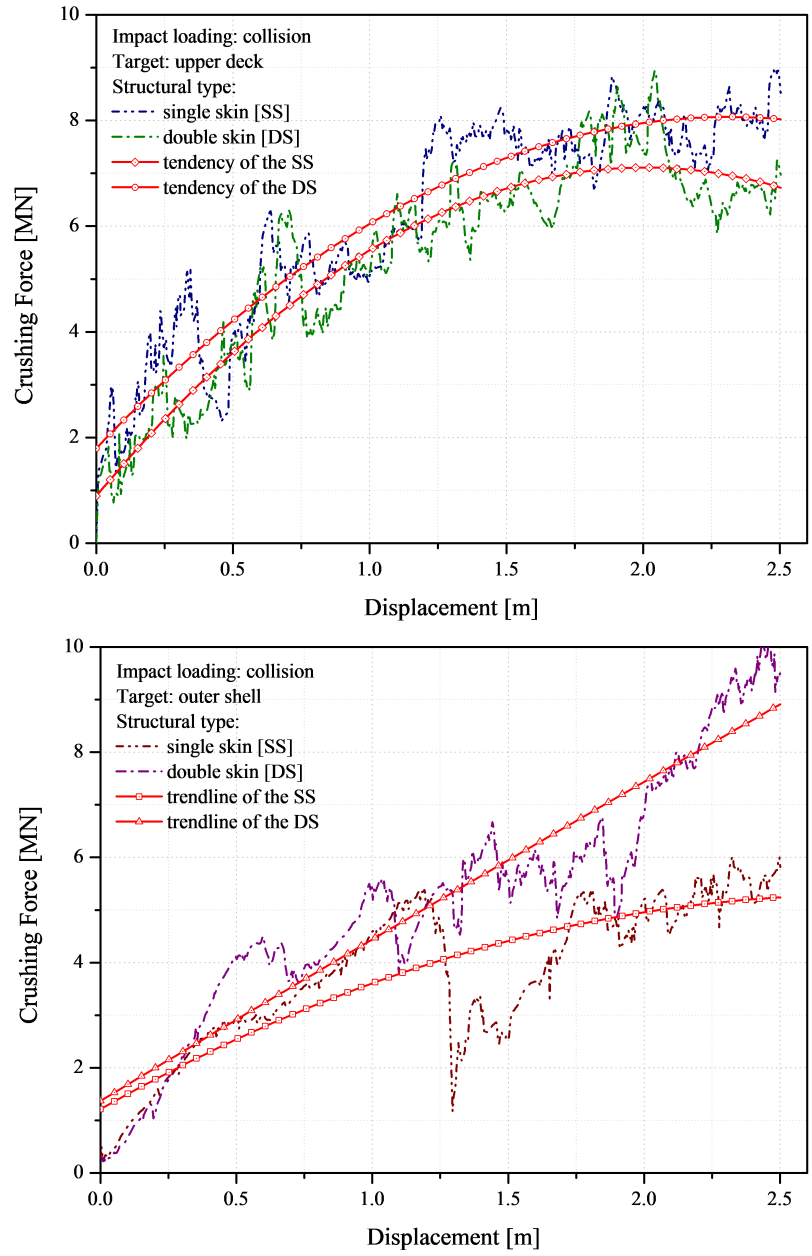

Figure 6: Crushing forces and their tendencies during collision

\subsection{Crushing force}

Resistance of the structure during the impact can be measured by quantifying the crushing force. This criterion is embodiment of structural fluctuation in experiencing impact. Since many elements/members are involved in such phenomenon, dynamic tendency is obtained in case of ship collision. In fact, however, tendency of the crushing force will be as similar as the absorbed energy.

This correlation is described in fundamental formulae of energy and force, respectively. As shown in Figure 6, it is obtained that tendency of the crushing force during contact to the upper deck is similar for SSS and DSS. Similar tendency is also shared during impact happened to the outer shell. The DSS indicated continuous increment, while the significant drop in force magnitude took place in collision to the SSS. After this phenomenon, the increment of the SSS's crushing force does not increase as high as the DSS due to inner structure of the SSS which can provide more resistance is no viable. 

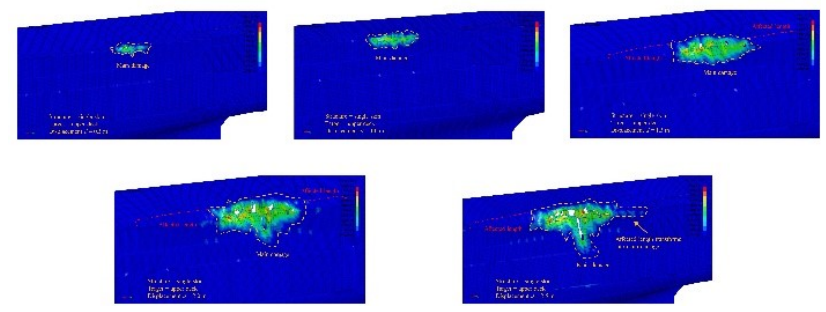

(a) Strain contours of the deck impact
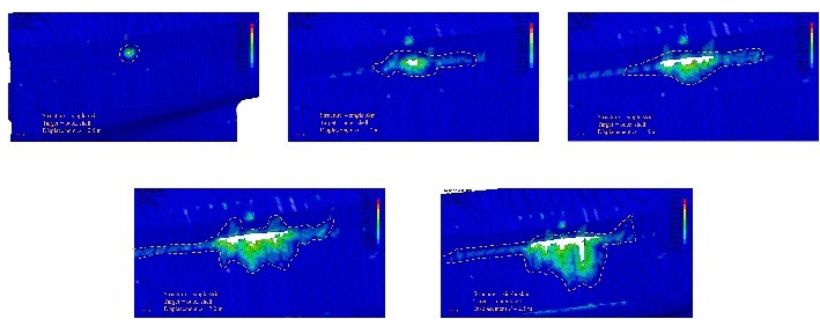

(b) Strain contours of the shell impact

Figure 7: Summary of progressive failure on the SSS

\subsection{Damage assessment}

Damage is an inevitable phenomenon which always occurs after an impact takes place, despite of its size will be small or large. This criterion is assessed in order to understand progressive damage contour on the designed SSS and DSS under the selected collision problems. The damage is classified in Figures 7 and 8 based on the structural types, SSS and DSS consecutively. During impact to the SSS's upper deck, the damage was found to be focused on the deck, and did not rapidly expand to lower structure, i.e. outer hull.

Indication of clear casualties on the outer hull occurred on displacement $D=1.5 \mathrm{~m}$, but only on the intersection area between upper deck and outer shell. However, in this very displacement distance, affected length took place as chain reaction of the structural rupture. This phenomenon later transformed to be main damage, which previously no strain contour was detected, but now it visible strain level can be observed in displacement $D=2.5 \mathrm{~m}$. In terms of the outer shell, results showed that rapid strain expansion occurred to the longitudinal direction. This indication is found since the displacement $D=1.0 \mathrm{~m}$, the strain contour occurred on the shell-stringer intersection, even though their intensity is still low. This contours was expanding, and its intensity was increasing in direction of the side stringer. Damage on the vertical direction is minor compared to the vertical direction. Findings related to the damage behavior on the SSS's deck are agree well with contours of the DSS during impacted to the deck. It is well
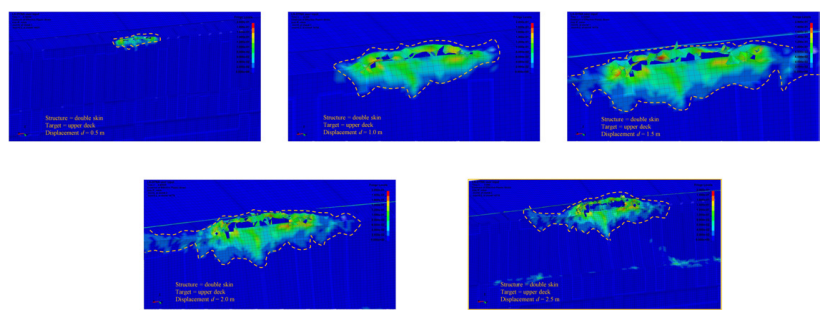

(a) Strain contours of the deck impact
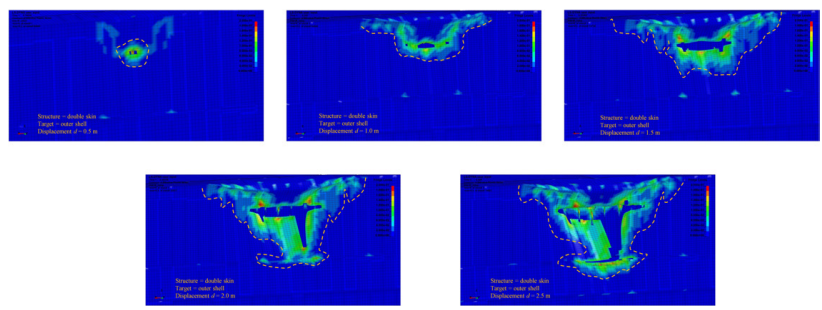

(b) Strain contours of the shell impact

Figure 8: Summary of progressive failure on the DSS

noted in Figure 8 that progressive contour on the deck was consider slow compared to the SSS's outer shell.

However, despite this similarity, contours to the vertical direction is quite different since tearing was reduced to certain extent so that vertical tearing did not occur until displacement $D=2.5 \mathrm{~m}$. Comparison in terms of the impact to the outer shell between SSS and DSS provided an conclusion opposite tendency. Previously, the outer shell which influenced by stringer geometry, progressed massively to the longitudinal direction. In other hand, the DSS's outer shell showed that major tearing occurred on the vertical direction which it reached interaction of the outer shell and middle deck. Summary of these discussions is quantified in form of damage length, width and affected length on the upper deck, and is presented in Appendix. Indication of damage progresses slower in during impact to the upper deck is verified by this summary, which the clear casualties appears on the upper deck later compared to the outer shell.

\subsection{Dynamic displacement}

Displacement of the structure is considered in this work to observe how far the striking ship pushed the side structures of the struck ship. The contours presented in Figures 9 and 10 stand for SSS and DSS consecutively, which is displacement contours on the transverse direction (Y axis). This direction is considered as it was selected axis for the striking ship's movement in scenario configuration. The displacement behavior of the SSS in experiencing impact 

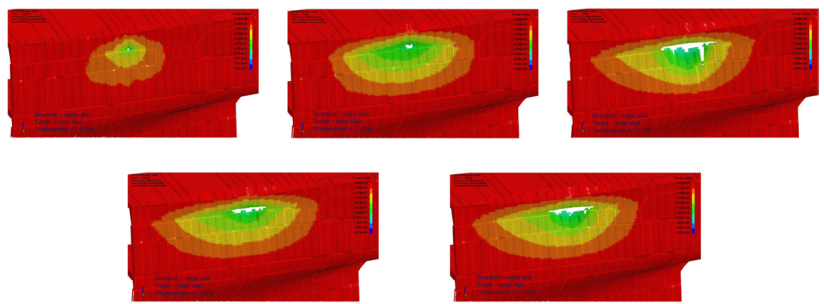

(a) Displacement contours of the upper deck
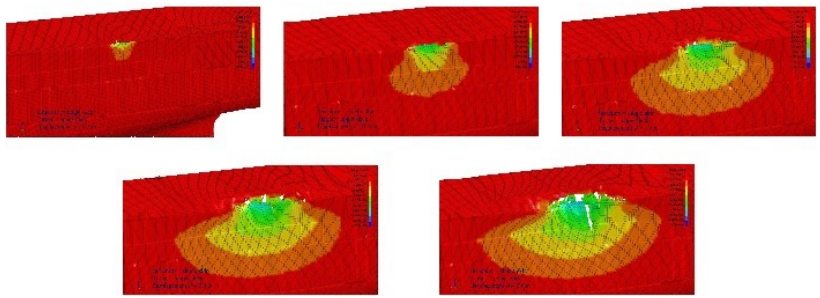

(b) Displacement contours of the outer shell

Figure 9: Summary of dynamic displacement behavior of the single side skin [SSS]
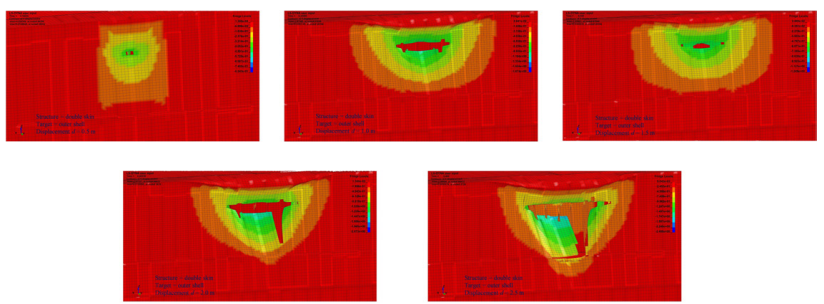

(a) Displacement contours of the upper deck
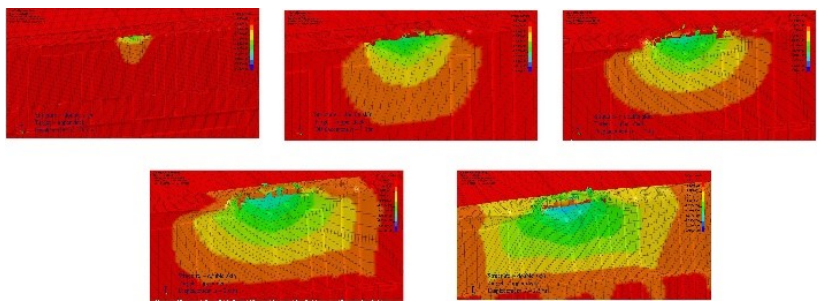

(b) Displacement contours of the outer shell

Figure 10: Summary of dynamic displacement behavior of the double side skin [DSS]

to the upper deck was more progressively expanding to the vertical direction compared to impact on the outer shell. Contours in deck impact formed a clear expansion directly to the vertical in displacement $D=1.0 \mathrm{~m}$, while the outer shell formed a circling contour surrounding the target.

Displacement contour of the SSS is found similar to deck impact of the DSS. Vertical direction is also the direction of the contour expansion which was already observed since displacement $D=0.5 \mathrm{~m}$. However, final form of the displacement contour is quite distinct as the DSS formed almost rectangular shape light green, yellow and
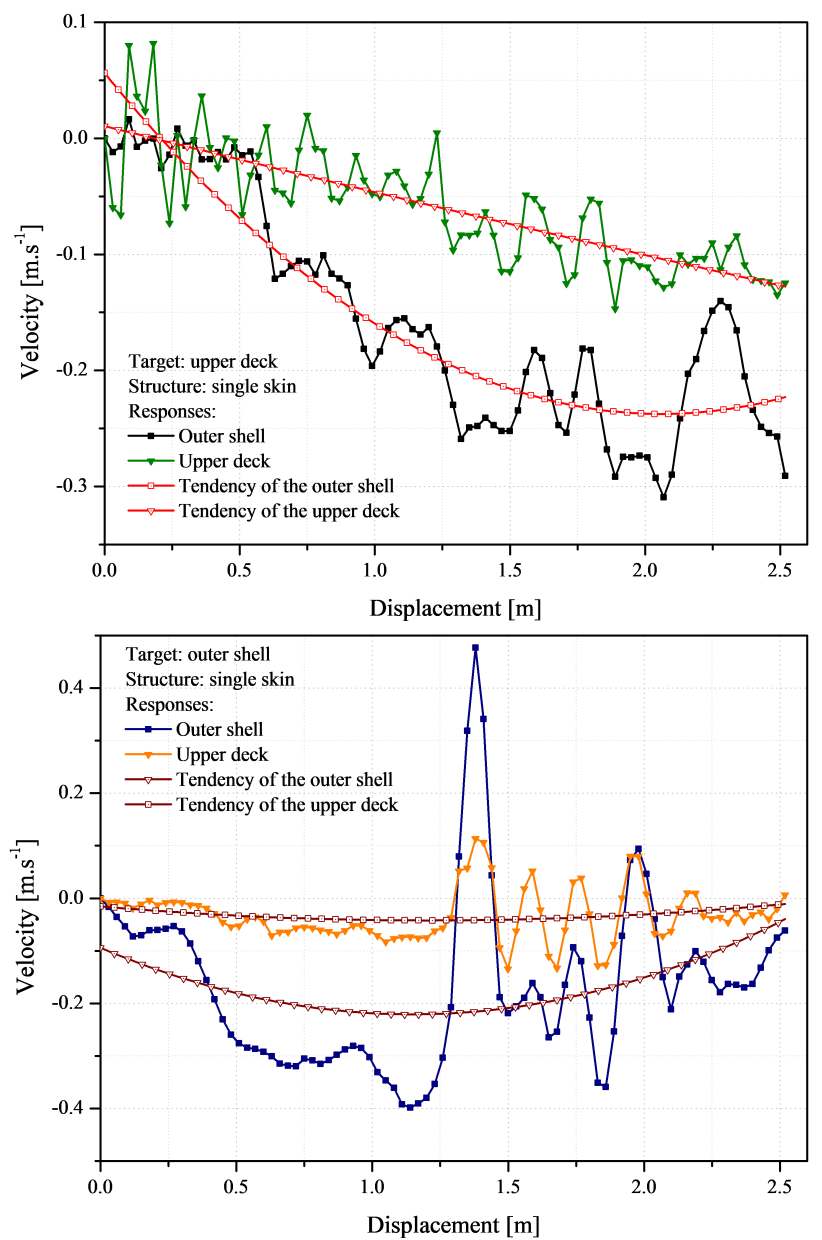

Figure 11: Velocity of the targets on single side skin [SSS] during side impact

orange contours, or approximately in member displacement 0.308-1.403 m. Significant disparity is observed on the DSS's shell compared to the SSS's which contours on the DSS clearly expanded mostly to vertical direction. In the end of collision process, it is also observed that the inner shell of the DSS has experienced displacement in range $0.245-0.495 \mathrm{~m}$.

\subsection{Member movement}

Velocity in this work is presented to observe movement behavior of the two selected members, i.e. upper deck and outer shell during impact loading is applied on two different structural types. The observed values represents their movement on the transverse direction ( $\mathrm{Y}$ axis), and minus mark indicates the movement is same as coming direction of the striking ship. Tendency of the SSS in Figure 11 concluded that the outer shell was the most moving member 

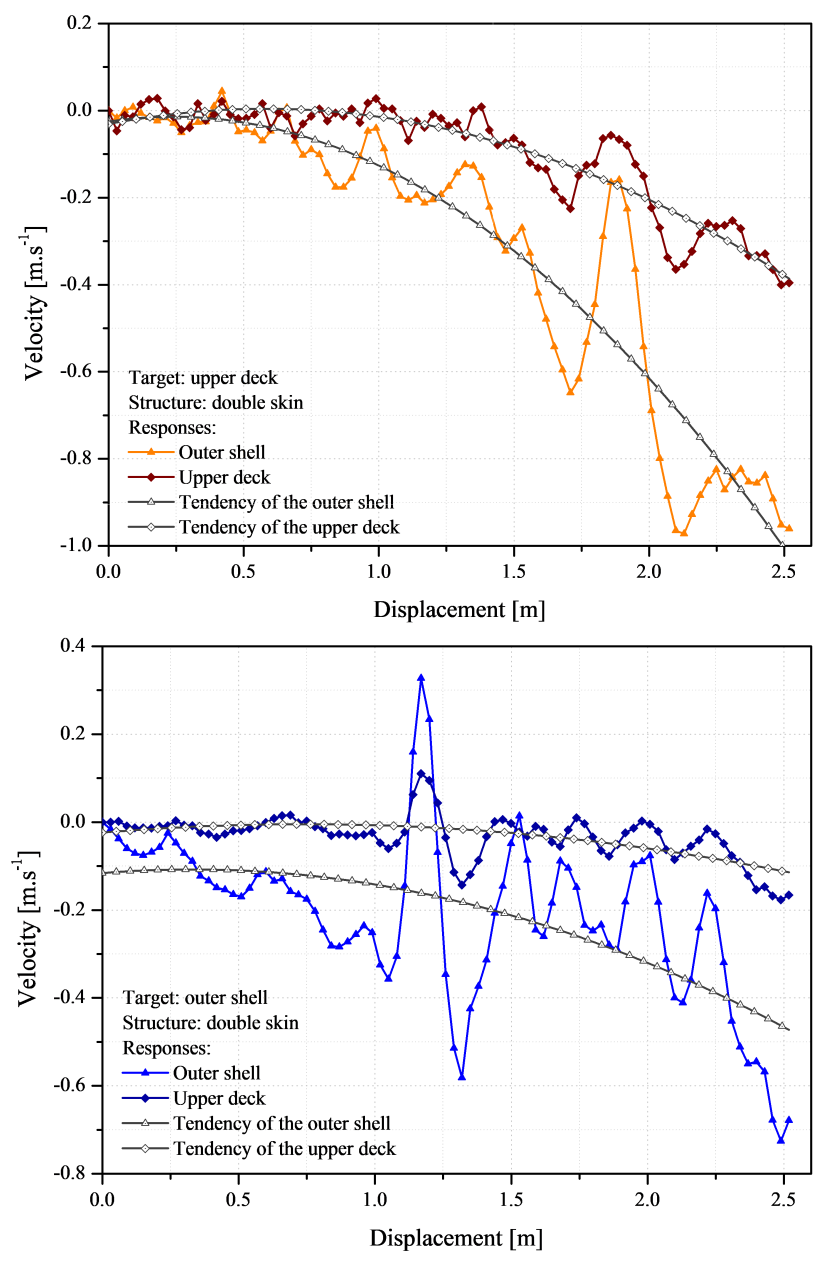

Figure 12: Velocity of the targets on double side skin [DSS] during side impact

despite of the target location was placed on the deck or shell. It is noted that behavior during target was selected to be on the upper deck, velocity fluctuation were gradually increasing as deeper penetration occurred on the deck. Distinct behavior is found during the target was moved to the outer shell. The upper deck during this impact tended to be more steady with quite high velocity value can be observed after the striking ship's displacement surpassed $D=1.25 \mathrm{~m}$. on the contrary, velocity of the outer shell is quite high since displacement $D=0.25 \mathrm{~m}$, which the value reached the peak approximately $D=1.25 \mathrm{~m}$. It is predicted that in this moment, the side stringer which supported the outer shell reached the ultimate strain and experienced rupture. In terms of the DSS (see Figure 12), impact to the upper deck produced similarity to the SSS's case which the gradual velocity of the members took place equals with penetration/displacement of the striking ship.

High increment in velocity occurred since displacement $D=1.5 \mathrm{~m}$ that marked tearing on the intersection of the deck and shell expanded to the lower member, i.e. outer shell which formed vertical tearing. In terms of the impact to the DSS's outer shell, the upper also showed passive movement in the initial part of penetration, or approximately until $D=1.0 \mathrm{~m}$. After this, the upper deck and outer shell experienced velocity increment as the two members were distorted and led to rupture which from the damage assessment in previous discussion, it is obtained that the longitudinal tearing on the shell is in $D=1.5 \mathrm{~m}$. Tearing on the outer hull made the shell continued to move as deeper as the striking ship penetration. Since the striking ship move in the $-Y$ axis, velocity value of the shell was also found to be in minus area. Difference is spotted between SSS's and DSS's outer shell in terms of the velocity, which the SSS's tended to be back to steady ( 0 velocity) in the end of collision and the velocity value was not as high as the DSS's. This phenomenon occurred since the tearing on the SSS appeared as longitudinal tearing, while the DSS had transverse tearing. Velocity value on the DSS can also be referred as flapping of the torn shell part during the striking ship's penetration.

\section{Concluding remarks}

This paper presented a series of investigation on ship structures during impact loading, collision, was applied on them. A benchmark study was conducted in order to confirm the validity of the current numerical methodology. Single side skin (SSS) and double side skin (DSS) were modeled as the representative of the structures, which several members would be selected as targets of the striking ship. Nonlinear dynamic behaviors of the global structures and the local members, such as upper deck and outer skin/shell has been discussed.

The results of several collision problems in this study indicated that capability of ship structures against impact loading was developed when the SSS was modified into the DSS. This statement was taken after evaluation on global behavior of the absorbed energy, which showed significant increment under side collision, especially during the target was selected on the outer shell. Increment of the structural capability against impact in this location is very essential in terms of cargo safety. When hazardous and dangerous commodities, such as nuclear, chemical compound, crude oil etc., are the carrier, resistance of the structure will depend mostly on the role of the outer shell. The findings of the absorbed energy was confirmed by tendency of the crushing force. The SSS experienced a drastic reduction in resistance capability after striking ship's dis- 
placement surpassed $1.25 \mathrm{~m}$, which in this moment side stringer was found rupture and caused drop in resistance until approximately $1 \mathrm{MN}$. On the other hand, steady fluctuation of the DSS was observed until the end of the collision that provided an indication good condition of the inner skin/shell.

After global responses were evaluated, influence of the striking ship's impact to local members, such as upper deck and outer shell were assessed. Strain contours and damage extent of the SSS's upper deck indicates larger vertical tearing occurred on it compared to the DSS's. It was also noted that even though the impact happened on the upper deck, side stringer of the SSS was also found rupture in the end of collision. Interestingly, the outer shell of the SSS and DSS produced different damage extent, which the SSS had damage mostly expanded to the longitudinal direction, while the DSS experienced major vertical tearing. Related to this phenomenon, it can be predicted that the DSS which has middle deck as the intermediate member between the upper deck and the cargo deck makes the tearing which is initiated between the upper and middle decks, tends to progressively expanded to lower structure due to tearing of the transverse frame. This concept is considered match on the SSS which due to influence of the side stringer, the strain contours grows by following path of the stringer on the outer shell. Furthermore, this behavior also affected the displacement contours, which the SSS has elliptic shape, while contours of the DSS expands to the vertical direction and almost form square shape. Tearing type on the outer shell was also influencing movement behavior of the ruptured member during the striking ships/ penetration. Velocity of the SSS's shell has smaller value compared to the DSS's which is indicated that torn outer shell still flapped forward-backward during the penetration.

Dynamic behaviors discussed in this study can be extended to the structural investigation on the hazardous carrier, such as chemical/oil tanker by considering previously occurred accident in the intersection routes. Consideration to factor of the incident location is encouraged in designing impact loading problem, which the conclusion of the further study can be used as realistic and dependable reference for the related authorities in designing shipping regulation for their water territories.

\section{References}

[1] Törnqvist R, 2003. Design of crashworthy ship structure. PhD thesis, Technical University of Denmark, Lyngby, Denmark. DOI:-

[2] Prabowo AR, 2018. Crashworthiness assessment of ship structures under collision and grounding. PhD thesis, Pukyong Na- tional University, Busan, South Korea. DOI:-

[3] Allianz, 2017. Safety and shipping review 2017; An annual review of trends and developments in shipping losses and safety. Allianz Global Corporate and Specialty, Munich, Germany. DOI:-

[4] Pedersen PT, Li Y, 2009. On the global ship hull bending energy in ship collisions. Marine Structures, 22, 2-11. DOI: https://doi. org/10.1016/j.marstruc.2008.06.005

[5] Gagnon RE, Wang J, 2012. Numerical simulations of a tanker collision with a bergy bit incorporating hydrodynamics, a validated ice model and damage to the vessel. Cold Regions Science and Technology, 81, 26-35. DOI: https://doi.org/10.1016/j. coldregions.2012.04.006

[6] Fan W, Yuan WC, 2014. Numerical simulation and analytical modeling of pile-supported structures subjected to ship collisions including soil-structure interaction. Ocean Engineering, 91, 1127. DOI: https://doi.org/10.1016/j.oceaneng.2014.08.011

[7] Liu B, Soares CG, 2016. Assessment of the strength of doublehull tanker side structures in minor ship collisions. Engineering Structures, 120,1-12. DOI: https://doi.org/10.1016/j.engstruct. 2016.04.011

[8] Prabowo AR, Bae DM, Sohn JM, Zakki AF, Cao B, Cho JH, 2017a. Effects of the rebounding of a striking ship on structural crashworthiness during ship-ship collision. Thin-Walled Structures, 115, 225-239. DOI: https://doi.org/10.1016/j.tws.2017.02.022

[9] Martinez JL, Cyrino JCR, Vaz MA, 2017. Continuum damage mechanics applied to numerical analysis of ship collisions. Marine Structures, 56, 206-236. DOI: https://doi.org/10.1016/j. marstruc.2017.08.003

[10] Collette M, Zhan Z, Zhu L, Zanic V, Okada T, Arima T, Skjong R, Jeong HK, Egorov G, 2018. Design principles and criteria. Proceedings of the 20th International Ship and Offshore Structures Congress (ISSC 2018), Volume I. DOI: 10.3233/978-1-61499-8624-549

[11] Youssef SAM, Paik JK, 2018. Hazard identification and scenario selection of ship grounding accidents. Ocean Engineering, 153, 242-255. DOI: https://doi.org/10.1016/j.oceaneng.2018.01.110

[12] ANSYS, 2019. ANSYS LS-DYNA User's Guide. ANSYS, Inc., Pennsylvania, US. DOI:-

[13] Allianz, 2012. Safety and Shipping 1912-2012, From Titanic to Costa Concordia; An insurer's perspective from Allianz Global Corporate \& Specialty. Allianz Global Corporate and Specialty, Munich, Germany. DOI:-

[14] Minosky VU, 1958. An analysis of ship collision with reference to protection of nuclear power ship. Journal of Ship Research, 3, 1-4. DOI:-

[15] Woisin G, 1979. Design against collision. Schiff \& Hafen, 31, 10591069. DOI:-

[16] Cao B, Bae DM, Sohn JM, Prabowo AR, Chen TH, Li H, 2016. Numerical analysis for damage characteristics caused by ice collision on side structure. Proceedings of the ASME 2016 35th International Conference on Ocean, Offshore and Arctic Engineering, 8, V008T07A019. DOI: http://dx.doi.org/10.1115/OMAE2016-54727

[17] Prabowo AR, Baek SJ, Cho HJ, Byeon JH, Bae DM, Sohn JM, 2017b. The effectiveness of thin-walled hull structures against collision impact. Latin American Journal of solids and Structures, 14, 13451360. DOI: http://dx.doi.org/10.1590/1679-78253895

[18] Obisesan A, Sriramula S, 2017. Performance characterisation for risk assessment of striking ship impacts based on struck ship damaged volume. Journal of Marine Science and Application, 16, 111-128. DOI: https://doi.org/10.1007/s11804-017-1403-0 
[19] Prabowo AR, Muttaqqie T, Sohn JM, Bae DM, 2018a. Nonlinear analysis of inter-island RoRo under impact: effects of selected collision's parameters on the crashworthy double-side structures. Journal of the Brazilian Society of Mechanical Sciences and Engineering, 40, 248. DOI: https://doi.org/10.1007/s40430018-1169-6

[20] Prabowo AR, Cho HJ, Lee SG, Baek SJ, Byeon JH, Bae DM, Sohn JM, Harsritanto BIR, 2018c. Evaluating structural crashworthiness and progressive failure of double hull tanker under accidental grounding: bottom raking case. Open Engineering, 8, 193-204. DOI: https://doi.org/10.1515/eng-2018-0024

[21] Zhang S, Pedersen PT, 2016. A method for ship collision damage and energy absorption analysis and its validation. Ships and Offshore Structures, 12, S11-S20. DOI: https://doi.org/10.1080/ 17445302.2016.1254584
[22] Zhang S, 1999. The mechanics of ship collisions. PhD thesis, Technical University of Denmark, Lyngby, Denmark. DOI:-

[23] Bae DM, Prabowo AR, Cao B, Zakki AF, Haryadi GD, 2016. Study on collision between two ships using selected parameters in collision simulation. Journal of Marine Science and Application, 15, 63-72. DOI: https://doi.org/10.1007/s11804-016-1341-2

[24] Ozguc O, Das PK, Barltrop N, 2005. A comparative study on the structural integrity of single and double side skin bulk carriers under collision damage. Marine Structures, 18, 511-547. DOI: https://doi.org/10.1016/j.marstruc.2006.01.004

[25] Prabowo AR, Sohn JM, Bae DM, Cho JH, 2018b. Estimating structure response and progressive failure of a ship hull under side-bow collisions. Tehnicki Vjesnik, 25, 1513-1522. DOI: https://doi.org/10.17559/TV-20170215113629 


\section{Appendix}

Table A.1: Summary of the damage assessment on the SSS: impact to the upper deck

\begin{tabular}{ccccc}
\hline No. & Displacement $[\mathrm{m}]$ & Longitudinal tearing $[\mathrm{m}]$ & Vertical tearing $[\mathrm{m}]$ & Affected length $[\mathrm{m}]$ \\
\hline 1 & 0 & 0 & 0 & 0 \\
2 & 0.5 & 0 & 0 & 2.2691 \\
3 & 1 & 0 & 0 & 5.2779 \\
4 & 1.5 & 2.9833 & 0.2777 & 10.7928 \\
5 & 2 & 3.0179 & 1.1156 & 12.4562 \\
6 & 2.5 & 4.2152 & 1.8308 & 13.1740 \\
\hline
\end{tabular}

Table A.2: Summary of the damage assessment on the SSS: impact to the outer shell

\begin{tabular}{ccccc}
\hline No. & Displacement $[\mathrm{m}]$ & Longitudinal tearing $[\mathrm{m}]$ & Vertical tearing $[\mathrm{m}]$ & Affected length $[\mathrm{m}]$ \\
\hline 1 & 0 & 0 & 0 & 0 \\
2 & 0.5 & 0.1467 & 0.1518 & 2.3928 \\
3 & 1 & 0.4959 & 0.2827 & 9.4559 \\
4 & 1.5 & 2.4247 & 0.4966 & 10.1763 \\
5 & 2 & 3.1541 & 0.7929 & 11.9745 \\
6 & 2.5 & 3.8915 & 1.6274 & 12.573 \\
\hline
\end{tabular}

Table A.3: Summary of the damage assessment on the DSS: impact to the upper deck

\begin{tabular}{ccccc}
\hline No. & Displacement $[\mathrm{m}]$ & Longitudinal tearing $[\mathrm{m}]$ & Vertical tearing $[\mathrm{m}]$ & Affected length $[\mathrm{m}]$ \\
\hline 1 & 0 & 0 & 0 & 0 \\
2 & 0.5 & 0 & 0 & 2.3876 \\
3 & 1 & 2.1663 & 0.3289 & 4.1795 \\
4 & 1.5 & 2.1969 & 0.4970 & 6.0657 \\
5 & 2 & 2.2219 & 0.9097 & 7.6846 \\
6 & 2.5 & 2.5408 & 1.0374 & 8.4074 \\
\hline
\end{tabular}

Table A.4: Summary of the damage assessment on the DSS: impact to the outer shell

\begin{tabular}{ccccc}
\hline No. & Displacement $[\mathrm{m}]$ & Longitudinal tearing $[\mathrm{m}]$ & Vertical tearing $[\mathrm{m}]$ & Affected length $[\mathrm{m}]$ \\
\hline 1 & 0 & 0 & 0 & 0 \\
2 & 0.5 & 0.2609 & 0.1166 & 2.3953 \\
3 & 1 & 0.7196 & 0.1859 & 5.8596 \\
4 & 1.5 & 2.3985 & 0.5594 & 6.9371 \\
5 & 2 & 3.1114 & 1.7706 & 7.1785 \\
6 & 2.5 & 3.7144 & 2.2368 & 7.4154 \\
\hline
\end{tabular}

